\title{
Delayed Granulomas as a Complication Secondary to Lip Augmentation with Dermal Fillers: A Systematic Review
}

\author{
Lily Nguyen Trinh, MD ${ }^{1,2}$ Kelly Christine McGuigan, $\mathrm{MS}^{3} \quad$ Amar Gupta, MD ${ }^{4}$
}

${ }^{1}$ Department of Otolarynology- Head and Neck Surgery, School of Medicine, Tulane University School of Medicine, New Orleans, Louisiana

2 Department of Otolaryngology-Head and Neck Surgery,

Massachusetts Eye and Ear Institution, Boston, Massachusetts

${ }^{3}$ Department of Otolarynology- Head and Neck Surgery, School of

Medicine, Kimmel Medical College, Thomas Jefferson University

Sidney, Philadelphia, Pennsylvania

${ }^{4}$ Department of Otolaryngology - Head and Neck Surgery, Facial

Plastic Surgery, Los Angeles, California

Surg J (NY) 2022;8:e69-e79.
Address for correspondence Lily Nguyen Trinh, MD, School of Medicine, Tulane University School of Medicine, New Orleans, Louisiana (e-mail: lilytrinh4@gmail.com).

\begin{abstract}
Keywords

- lip augmentation

- fillers

- nodules

- granulomas

- systematic review
\end{abstract}

Introduction Lip augmentation with dermal filler is rising in popularity. There are generally minimal side effects that are mild and transient. However, long-term complications may occur and include lumps, bumps, nodules, or granulomas. To better understand this uncommon but challenging outcome, we aim to perform a thorough systematic review of the published literature related to nodule or granuloma formation after cosmetic soft tissue augmentation of the lips.

Methods A search of published literature was conducted in accordance with PRISMA (Preferred Reporting Items for Systematic Reviews and Meta-Analyses) guidelines in April 2021 and included PubMed, ScienceDirect, Embase, Google Scholar, and Cochrane databases. The Medical Subject Headings (MeSH) terms used included the following terms: "lip filler," "hyaluronic acid," “lip injection," "lip augmentation," "silicone," "poly-L-lactic acid," "calcium hydroxyapatite," "polymethylmethacrylate," "complications," "reaction," "granuloma," and "nodule." All studies were reviewed by two independent reviewers. Any discrepancies were resolved by a third reviewer.

Results The initial search for filler-related nodules or granulomas yielded 2,954 articles and 28 were included in the final analysis containing 66 individual cases of lip nodules. All but one patient was female. The mean age was 50 years. Nodules presented on average 35.2 months or 2.9 years after initial treatment. Thirty-seven nodules underwent histological analysis, the majority of which identified the presence of a foreign-body granuloma. Silicone was the most reported filler used followed by hyaluronic acid. Most cases resolved following multiple treatments including oral antibiotics or steroids followed by surgical excision.

Conclusion Understanding the sequelae of lip augmentation with filler products allows clinicians to provide safe and effective treatment. Nodules that present months to years following dermal treatment may represent a foreign-body granuloma. A combination of oral antibiotics, intralesional or oral steroids, and surgical excision successfully treated the majority of cases in our study. received

October 21, 2021 accepted after revision January 20, 2022
DOI https://doi.org/

$10.1055 / \mathrm{s}-0042-1743524$

ISSN 2378-5128. (c) 2022. The Author(s).

This is an open access article published by Thieme under the terms of the Creative Commons Attribution License, permitting unrestricted use, distribution, and reproduction so long as the original work is properly cited. (https://creativecommons.org/licenses/by/4.0/)

Thieme Medical Publishers, Inc., 333 Seventh Avenue, 18th Floor, New York, NY 10001, USA 
The lips are a central and defining aspect of an individual's face and have long been a target for enhancement and antiaging techniques. Due to the multitude of treatment modalities available today, treatments to the lips are becoming increasingly more common. There are numerous materials for lip augmentation including hyaluronic acid (HA), fat grafts, silicone, polyacrylamide, polymethylmethacrylate (PMMA), and poly-L-lactic acid (PLLA). Injections are used to treat lip asymmetry, lack of vermillion volume, vertical lip lines, downturned oral commissures, and/or an elongated upper lip, features which are normal consequences of aging. ${ }^{1}$ Lip enhancement or augmentation with injectable fillers is rising in popularity as these treatments achieve rapid results with generally predictable outcomes. ${ }^{2}$ This aesthetic treatment is also favorable due to its less invasive nature and minimal down time compared with surgical cosmetic procedures.

Soft tissue fillers are generally well-tolerated and major adverse events are rare. Mild side effects typically include limited and transient swelling, bruising, pain, and erythema. More severe complications include infection, nodule formation, vascular occlusion, and pigment changes. ${ }^{3}$ Nodule formation may be characterized as early or delayed ( 4 weeks and later postinjection). ${ }^{4}$ The incidence of delayed-onset nodules is uncommon and is reported to be 0.1 to $1.0 \%{ }^{5}$ Delayed-onset nodules may be identified through histological analysis as foreign-body granulomas. If nodules or granuloma formation occur, they can be treated with intralesional steroids or hyaluronidase injections (for HA filler). However, if these treatments repeatedly fail, surgical excision may be required.,

Delayed-onset granuloma formation has been a rarely cited complication of lip augmentation with filler. In this review, we aim to perform a thorough systematic review of the published literature related to nodule or granuloma formation after lip filler injections. Our goal is to determine the details associated with this complication including symptom description, time of onset, nodule characteristics, treatment, and outcomes. Since various terms for nodular formations have been used across providers, including lumps, bumps, nodules, or granulomas, these terms will be interchangeably used throughout this study unless otherwise specified. By gaining a better understanding of this rare but challenging outcome of lip fillers, providers can prevent future complications and provide patients reliable information regarding potential side effects.

\section{Methods}

\section{Search Strategy}

A systematic review of the published literature was conducted in accordance with the Preferred Reporting Items for Systematic Reviews and Meta-Analyses (PRISMA) guidelines to assess the association of granulomas with HA lip fillers. The literature search was performed in April of 2021, which included PubMed, ScienceDirect, Embase, Google Scholar, and Cochrane databases. The Medical Subject Headings (MeSH) terms used included the following terms: "lip filler," "hyaluronic acid," “lip injection,” "lip augmentation,” “silicone," “poly-L-lactic acid," "calcium hydroxyapatite," "polymethylmethacrylate," "complications," "reaction," "granuloma," and "nodule." The goal of the search was to compile and assess all of the published literature consisting of original articles including case reports, clinical trials, case series, and prospective case studies related to granuloma formation after filler lip augmentation.

\section{Study Selection}

Studies were included if they met the following criteria: (1) described granuloma formation after lip filler, (2) were published between 2000 and 2021, and (3) included specific patient case information. Exclusion criteria included studies that: (1) were not published in English, (2) included nonhuman subjects, (3) were abstracts, communications, letter to the editor, or review articles, (4) did not report on location, onset, and treatment of granuloma, and (6) those discussing granulomas in other areas of the face.

\section{Data Abstraction}

Titles and abstracts were screened for relevance by two separate reviewers (L.N.T. and K.C.M.). Of the selected articles that met the predetermined criteria, the full-text articles were retrieved and then independently reviewed by the two reviewers. Any discrepancies were resolved with the third investigator (A.G.). All studies which met the predetermined criteria were included in the final analysis. Relevant information from each included article was extracted which included author name, year of case reporting, patient age and sex, patient comorbidities, filler brand used, volume injected, site of injection, time of symptom onset after injection, presence of swelling or pain, number of nodules, nodule description, nodule duration/size, excision technique, treatment, and outcome. A summary of extracted information is depicted in -Table $\mathbf{1}$.

\section{Results}

\section{Study Selection}

The initial search for filler-related nodules or granulomas yielded 2,955 articles. After removing 1,016 duplicates, 1,939 studies were screened by title and abstract. A total of 1,852 studies were eliminated based on the predetermined inclusion and exclusion criteria. Five articles were unable to be retrieved. The remaining 82 articles underwent full-text review. Fifty-three studies were excluded due to wrong treatment area of the face $(n=17)$, do not include specific case information $(n=14)$, wrong study design $(n=10)$, wrong outcomes $(n=2)$, abstract only $(n=6)$, and published in a language other than English $(n=4)$. A total of 29 articles were included in the final analysis. - Fig. 1 demonstrates the breakdown of the literature search.

\section{Study Characteristics}

An overview of the study characteristics is summarized in -Table 1 for HA and in -Table 2 for non-HA. Of the 29 included studies, there were 68 cases reported on the incidence of nodule or granuloma formation following lip 


\begin{tabular}{|c|c|c|c|c|c|c|c|c|c|c|c|c|c|c|c|}
\hline 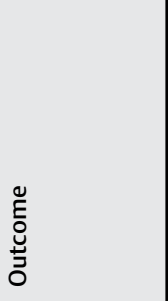 & 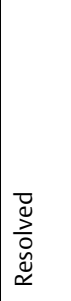 & 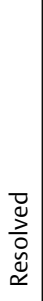 & 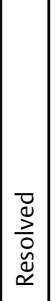 & 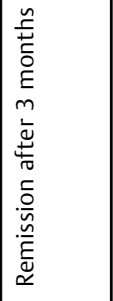 & 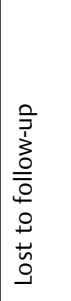 & 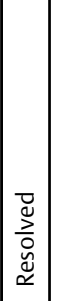 & 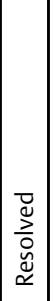 & 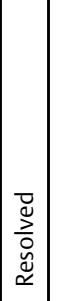 & 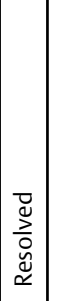 & 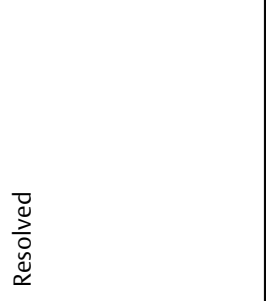 & $\Sigma$ & 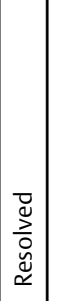 & $\Sigma$ & $\Sigma$ & 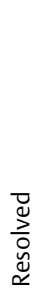 \\
\hline 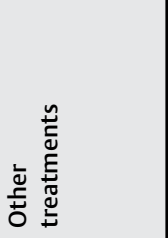 & $\Sigma$ & $\underline{z}$ & $\S$ & 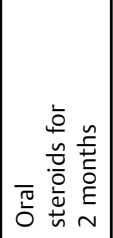 & $\underline{z}$ & $\Sigma$ & 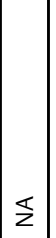 & 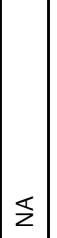 & \$ & 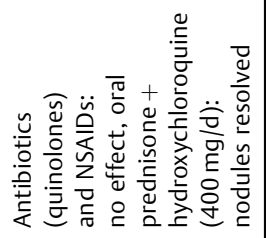 & $\Sigma$ & $\Sigma$ & $\Sigma$ & $\S$ & $\Sigma$ \\
\hline 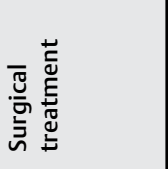 & $\begin{array}{l}\frac{.}{0} \\
: \frac{\bar{y}}{\bar{x}} \\
\dot{x}\end{array}$ & 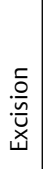 & 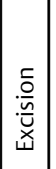 & 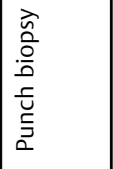 & 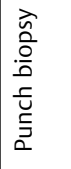 & 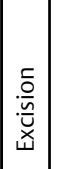 & 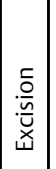 & 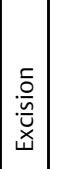 & \begin{tabular}{|c|}
$\frac{.}{0}$ \\
$: \frac{0}{\breve{y}}$ \\
$\dot{x}$ \\
\end{tabular} & 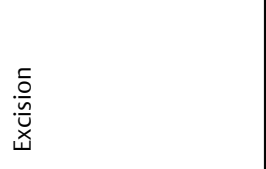 & $\S$ & 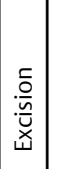 & $\Sigma$ & $\Sigma$ & 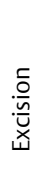 \\
\hline 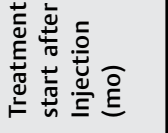 & $\stackrel{ \pm}{\sim}$ & $\Sigma$ & $\stackrel{m}{\mid}$ & $\simeq$ & $\sim$ & 0 & $\nabla$ & $\stackrel{\sim}{\sim}$ & $\frac{1}{z}$ & $\stackrel{\text { ㅇ }}{\llcorner}$ & 0 & $\stackrel{m}{|c|}$ & $\Sigma$ & $\Sigma$ & $\stackrel{m}{m}$ \\
\hline 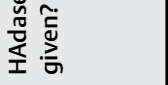 & z & z & 2 & $\stackrel{\check{\Perp}}{\rightleftharpoons}$ & z & 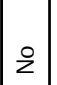 & 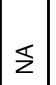 & $\Sigma$ & $\Sigma$ & z & 2 & $\stackrel{\check{\nu}}{\rightleftharpoons}$ & $\stackrel{2}{z}$ & 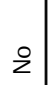 & $\Sigma$ \\
\hline 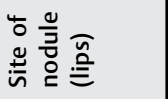 & 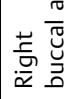 & 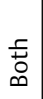 & 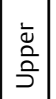 & 袬 & 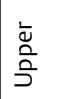 & 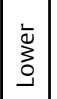 & 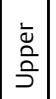 & 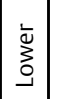 & 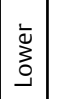 & 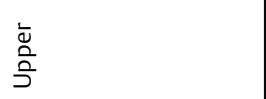 & 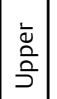 & 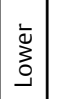 & 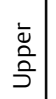 & 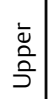 & 产 \\
\hline 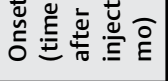 & $\stackrel{\mathbb{N}}{\sim}$ & $\simeq$ & 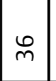 & $\underset{\leftarrow}{\stackrel{\Xi}{*}}$ & $\sim$ & 0 & $\nabla$ & $\stackrel{\sim}{\sim}$ & $\Sigma$ & $\stackrel{\llcorner}{\leftarrow}$ & 0 & 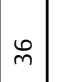 & $\Sigma$ & $\bar{z}$ & - \\
\hline 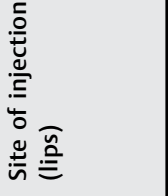 & 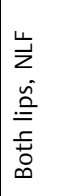 & 善 & 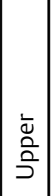 & 产 & 产 & 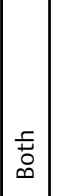 & 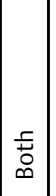 & $\mid$ & 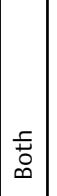 & 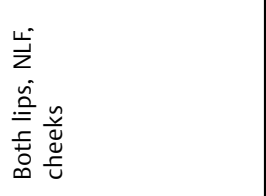 & 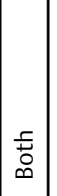 & $\mid$ & $\begin{array}{l}\grave{\grave{o}} \\
\hat{\mathrm{O}}\end{array}$ & 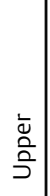 & 镸 \\
\hline $\begin{array}{l}\text { D } \\
\text { 兽 } \\
\text { D. }\end{array}$ & 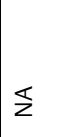 & 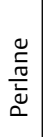 & $\Sigma$ & 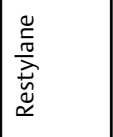 & 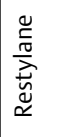 & 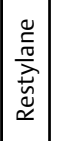 & 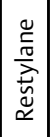 & 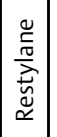 & 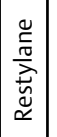 & 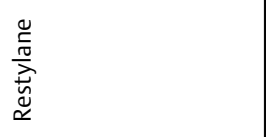 & 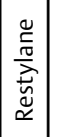 & 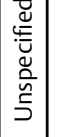 & $\Sigma$ & $\Sigma$ & 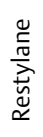 \\
\hline$\stackrel{㐅}{~}$ & 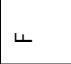 & $\leftarrow$ & 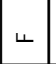 & 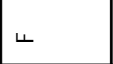 & \llcorner & 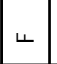 & 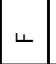 & \llcorner & \llcorner & 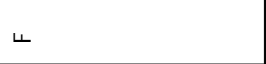 & \llcorner & $\leftarrow$ & \llcorner & 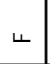 & \llcorner \\
\hline 娄 & ก & in & $\approx$ & ถ & $\stackrel{\infty}{+}$ & \ & in & in & in & f & in & $\approx$ & \pm & $\eta$ & ก \\
\hline $\begin{array}{l}\text { 辛 } \\
\text { 壳 }\end{array}$ & $\begin{array}{l}\text { 吾 } \\
\text { 范 } \\
0\end{array}$ & 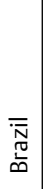 & 祘 & 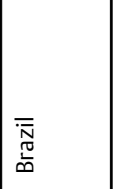 & 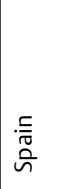 & 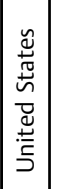 & 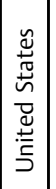 & & & $\begin{array}{l}\cdot \frac{5}{\overline{0}} \\
\hat{n} \\
\end{array}$ & & 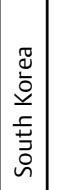 & 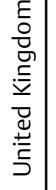 & & $\begin{array}{l}\frac{\pi}{0} \\
\text { 离 } \\
\text { v }\end{array}$ \\
\hline 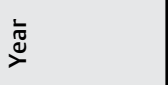 & 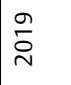 & $\stackrel{\tilde{D}}{i}$ & $\begin{array}{l}n \\
\stackrel{n}{2} \\
\end{array}$ & $\stackrel{n}{\grave{2}}$ & ڤ̊̀े & 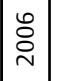 & $\begin{array}{l}n \\
\stackrel{2}{2}\end{array}$ & & & $\stackrel{m}{i}$ & & $\bar{\sim}$ & $\stackrel{\infty}{\grave{N}}$ & & 完 \\
\hline 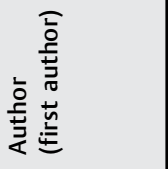 & 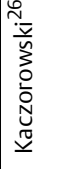 & 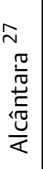 & 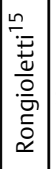 & . & 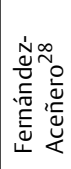 & 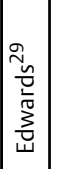 & 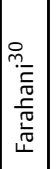 & & & 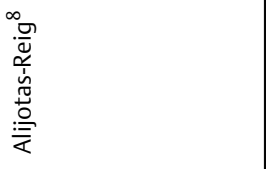 & & 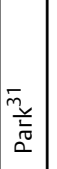 & & & 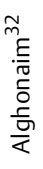 \\
\hline
\end{tabular}




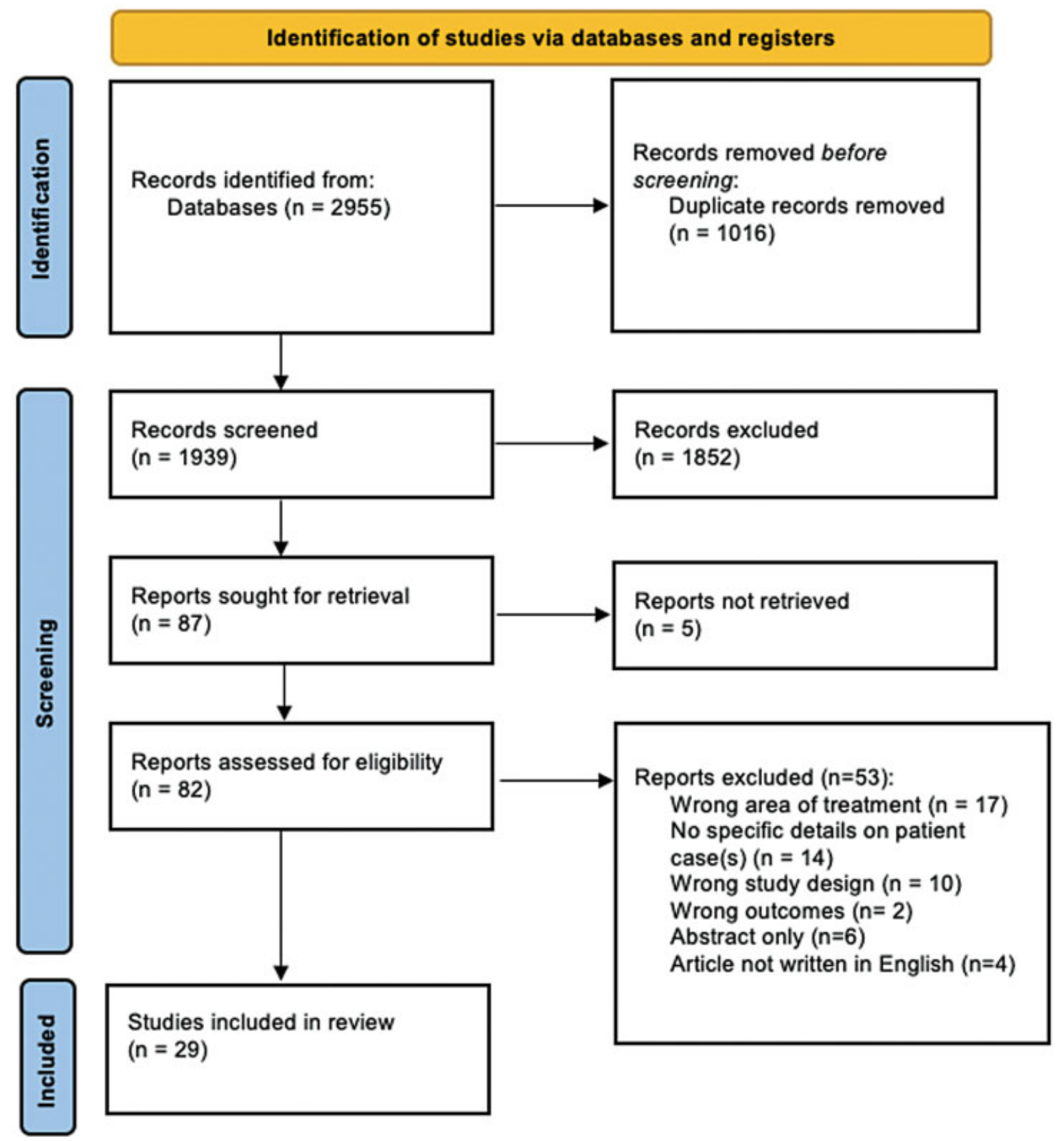

Fig. 1 PRISMA 2020 flow diagram of screening process.

augmentation with dermal filler. This selection of 29 articles included 15 (51.7\%) case reports (articles containing the description of one case) and 14 (48.3\%) case series (articles evaluating more than one case). Most studies originated from Spain ( $n=6 ; 20.7 \%)$. Four were from the United States, and many other countries were also represented (-Fig. 2). The article publication years ranged from 2003 to 2019 (-Fig. 3).

\section{Case Characteristics}

There were 67 (98.5\%) females and one (1.5\%) male described in our cases. The average age was 50.0 years (range: 23-77 years). The mean time of onset of masses or nodules was 34.4 $(n=55)$ months. Most patients presented with swelling, asymmetry, or erythema of their lips. Twenty-six cases
(38.2\%) presented with multiple nodules, 19 (27.9\%) reported single nodules, and 23 cases (33.8\%) did not report on the number of nodules. Masses were frequently described as discrete, indurated, mobile, firm, and slowly growing.

A histological analysis of 37 cases was reported. Thirtyone $(83.8 \%)$ of these confirmed a foreign-body granuloma. The other cases reported extensive or chronic inflammation, sarcoid-like reaction, and a pseudocystic, fibrous-structurecontaining translucent, viscous material that stained positive for Alcian blue (a marker for HA).

Only one case reported on the injection volume, which was $3 \mathrm{~mL}$ for both lips. ${ }^{7}$ No studies reported on the method of injection or qualifications of the injector. Regarding the initial treatment area for augmentation, many studies 


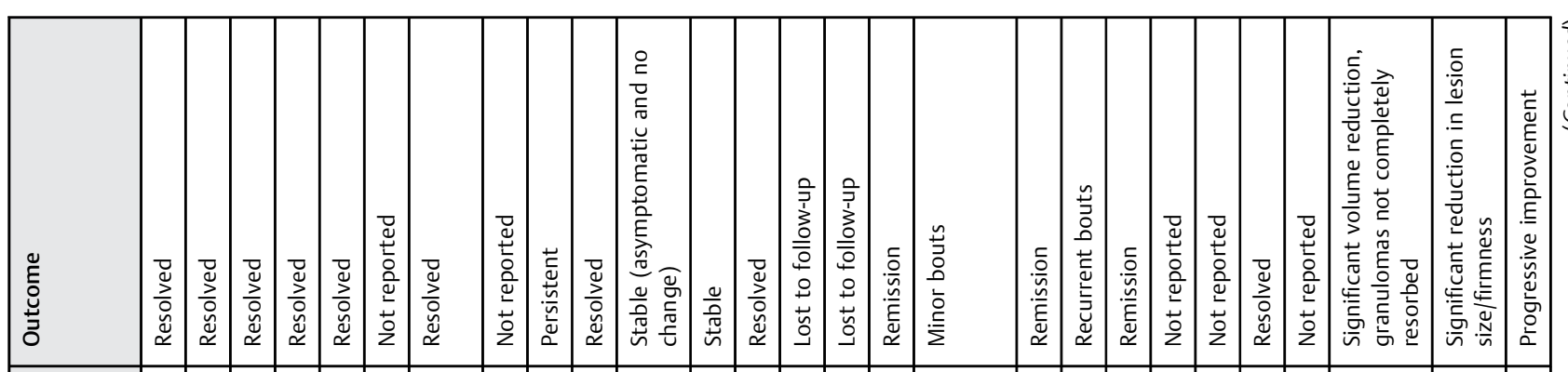

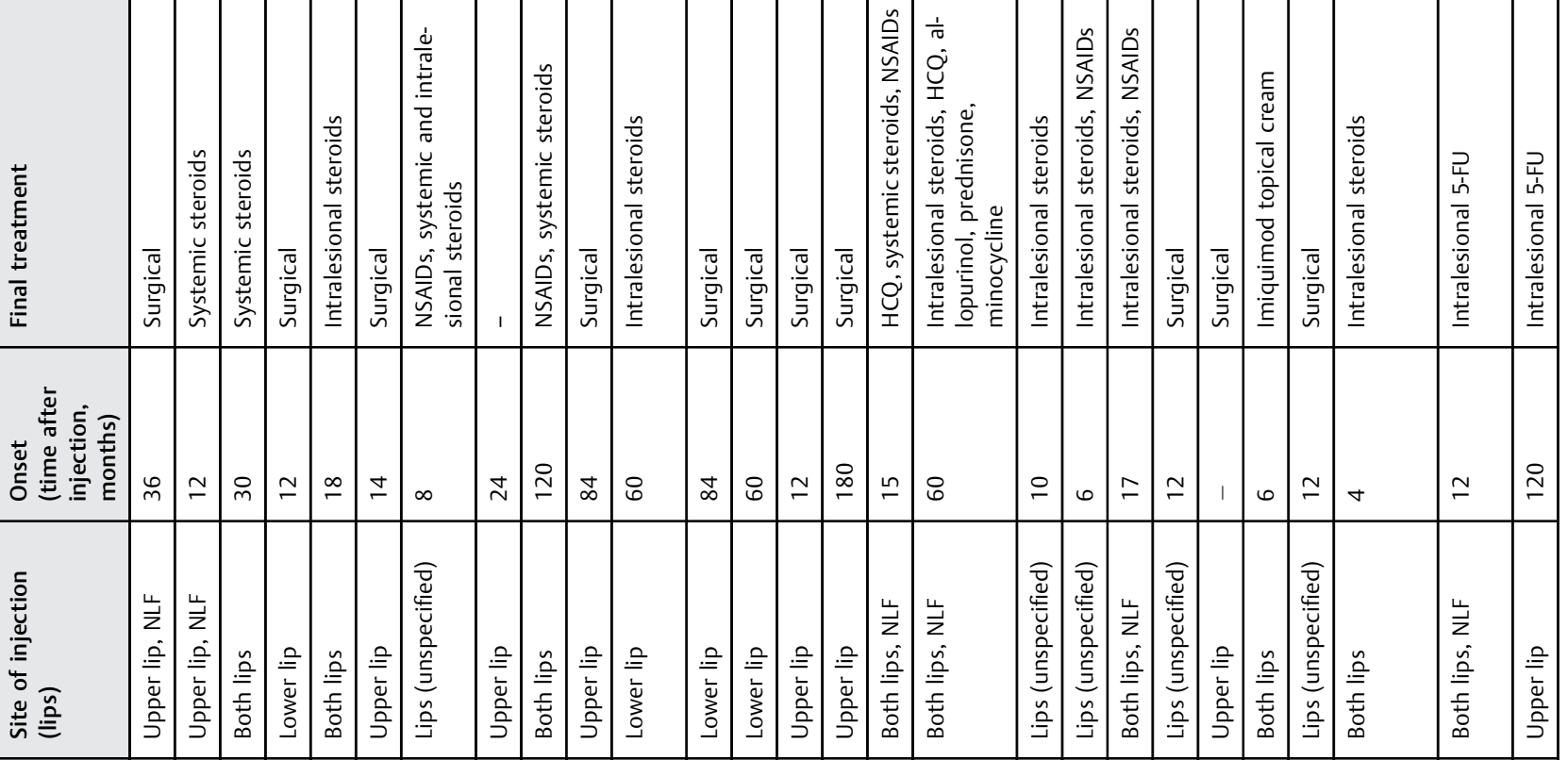

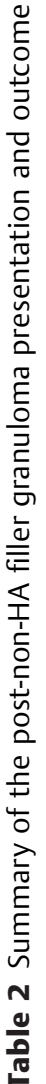

竞

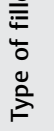

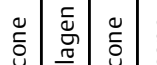

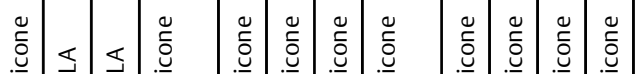

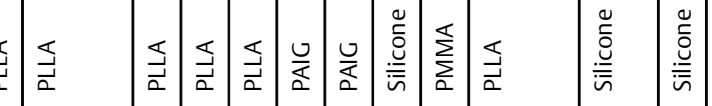

๗ั㐅

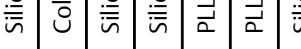

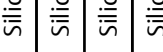

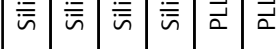

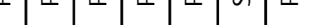

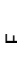

安

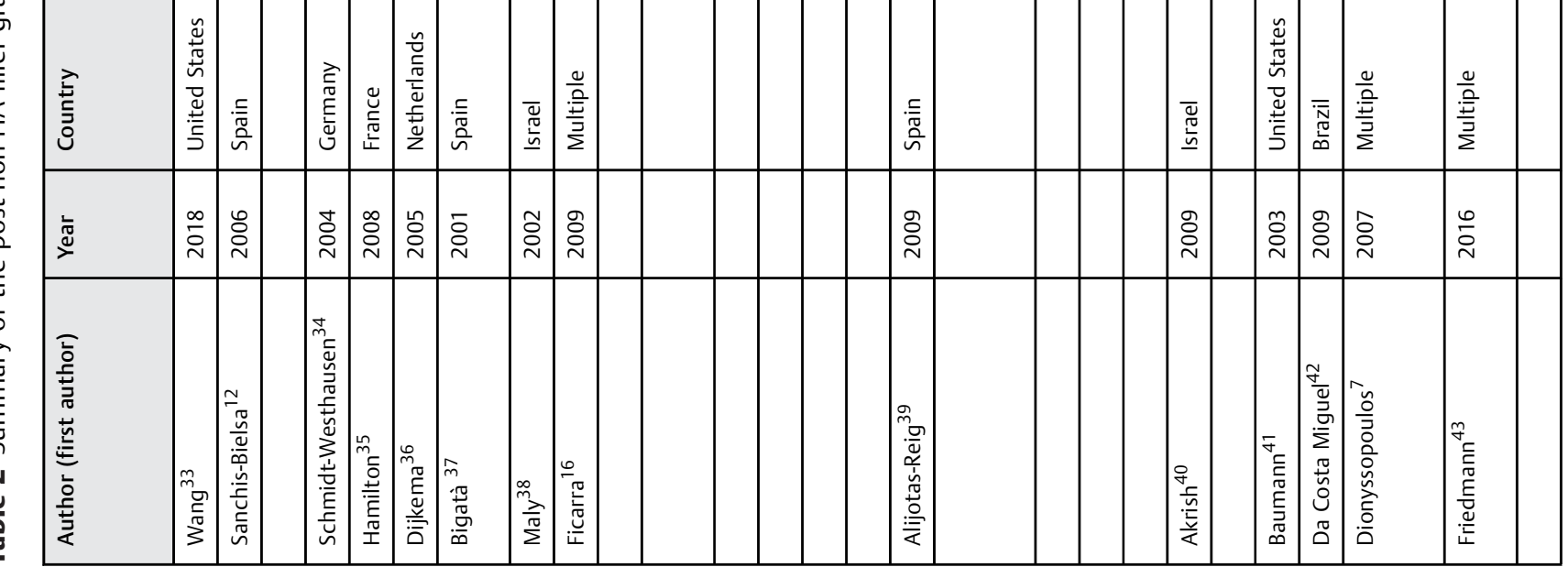




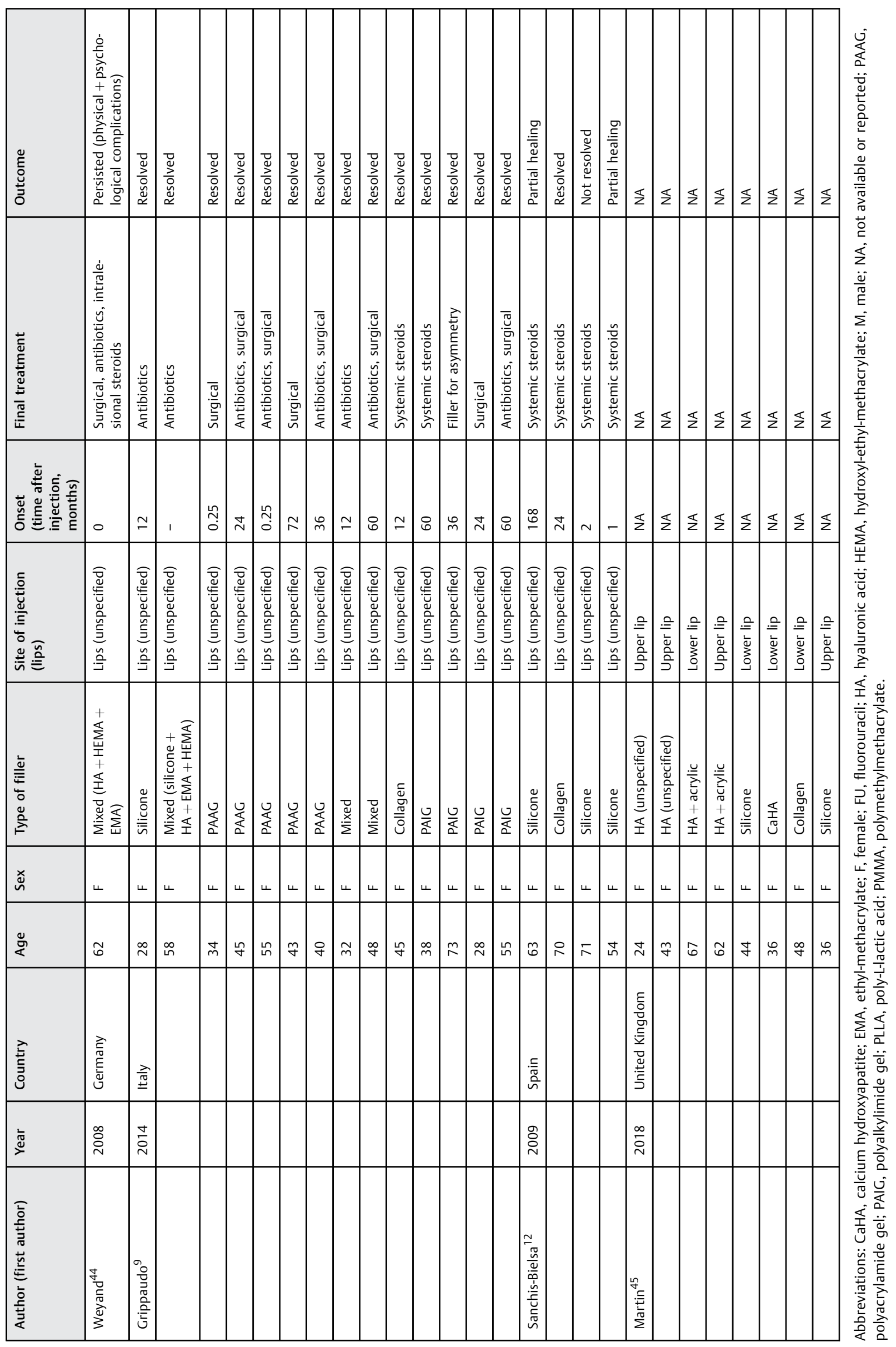




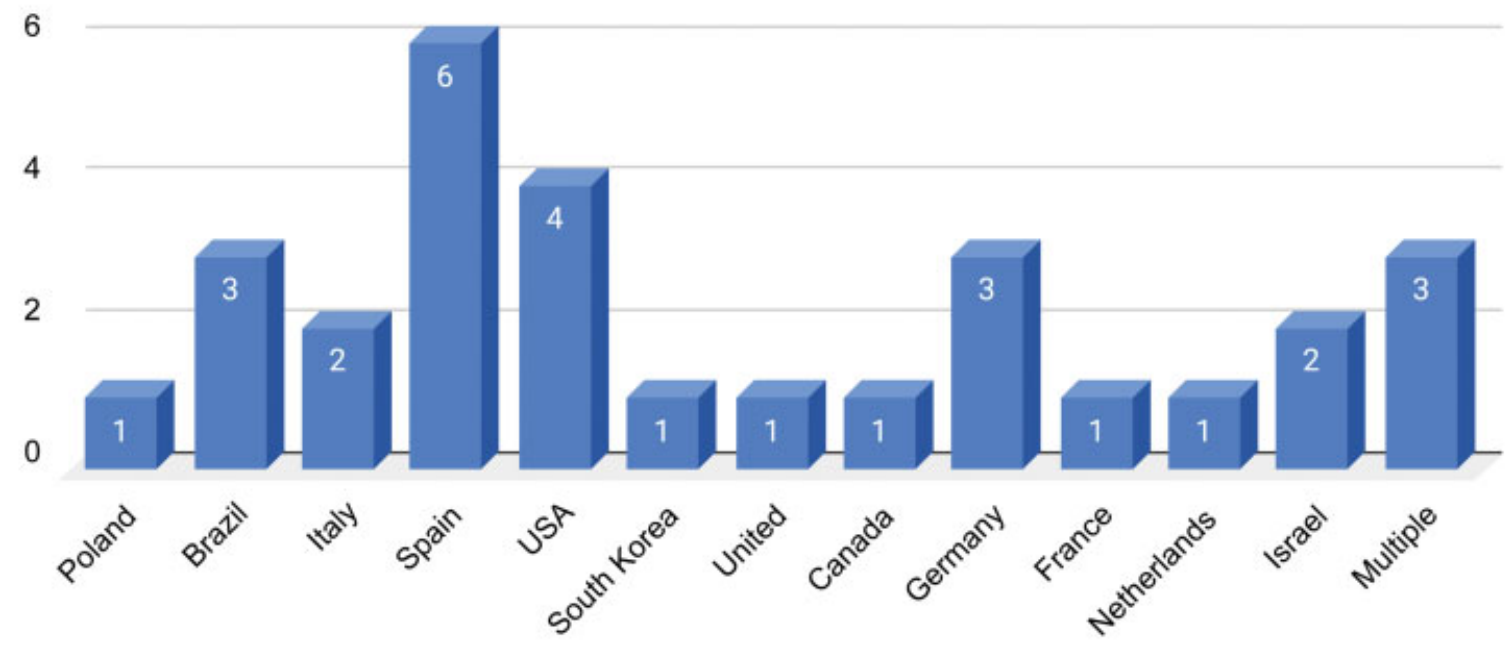

Fig. 2 Country of study publication.

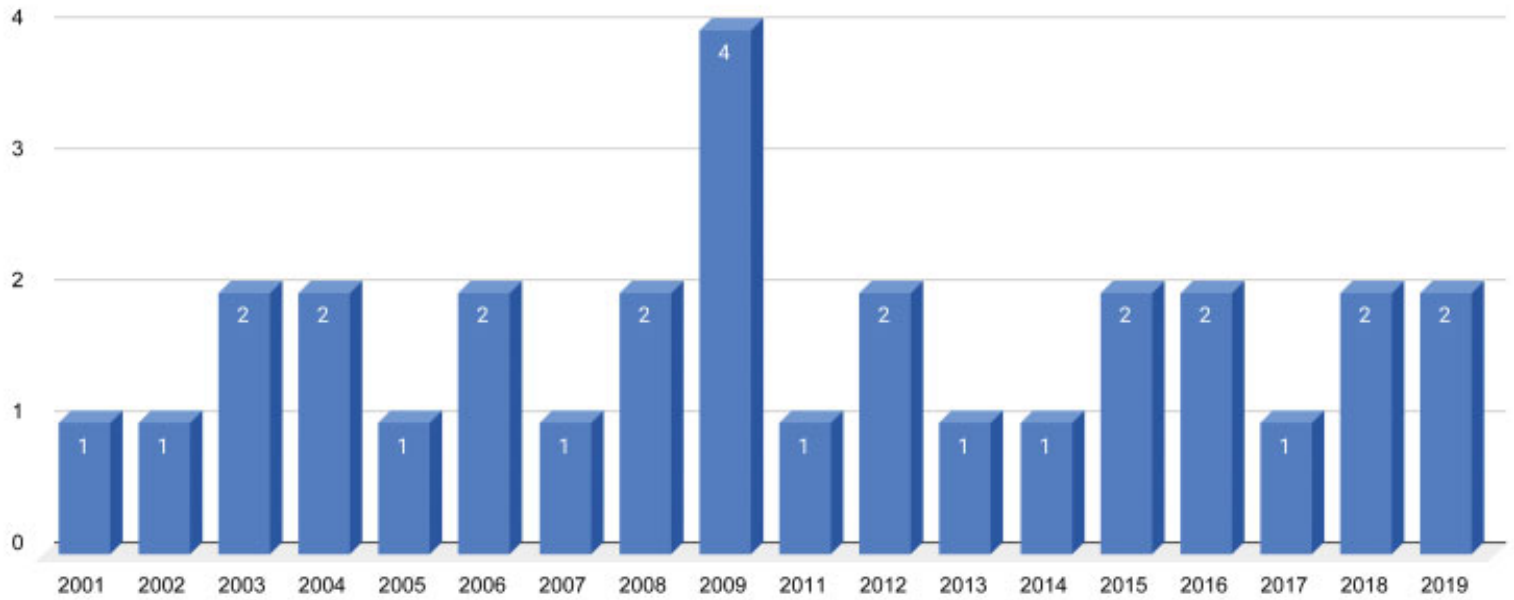

Fig. 3 Number of study publication by year.

reported injecting in the "lips" and did not specify if it was both lips and one lip. The frequency of other injected areas is displayed in - Fig. 4.

\section{Product Used}

The most commonly reported dermal filler product used was silicone $(21 / 66,31.8 \%)$ followed by HA (14/66, 21.2\%). Various other dermal fillers were used (-Fig. 5 ).

\section{Treatment}

Treatment typically consisted of oral antibiotics, intralesional steroids, or oral steroids (-Fig. 6). For instance, Alijotas-Reig et al initially treated a case of multiple nodules with antibiotics (quinolones) and nonsteroidal anti-inflammatory drugs (NSAIDs), which had no effect. ${ }^{8}$ However, when oral prednisone and hydroxychloroquine (400 $\mathrm{mg}$ /day) were added, many of the nodules resolved. Grippaudo et al described a case with "multiple angry red lumps" 12 months following lip augmentation, which was successfully treated with three rounds of antibiotics. ${ }^{9}$ Curi et al reported a non-well-defined nodule which was initially evaluated by a punch biopsy. This identified the foreign granuloma which was successfully treated with oral steroids for 2 months. ${ }^{10}$ Goldman and Wollina reported granulomas after PMMA injection which was first treated by intralesional $1,064 \mathrm{~nm} \mathrm{Nd}$ :YAG laser in combination with suction using a blunt liposuction cannula either alone or combined with surgery. ${ }^{11}$ Sanchis-Bielsa et al described a nodule with associated swelling that partially resolved with oral steroids (30-90 mg/day) for 10 to 15 days. ${ }^{12}$ Surgical treatment was often offered to those with persistent nodules (25/66, 37\%), which led to complete resolution of the nodule. 
25

20

15

10

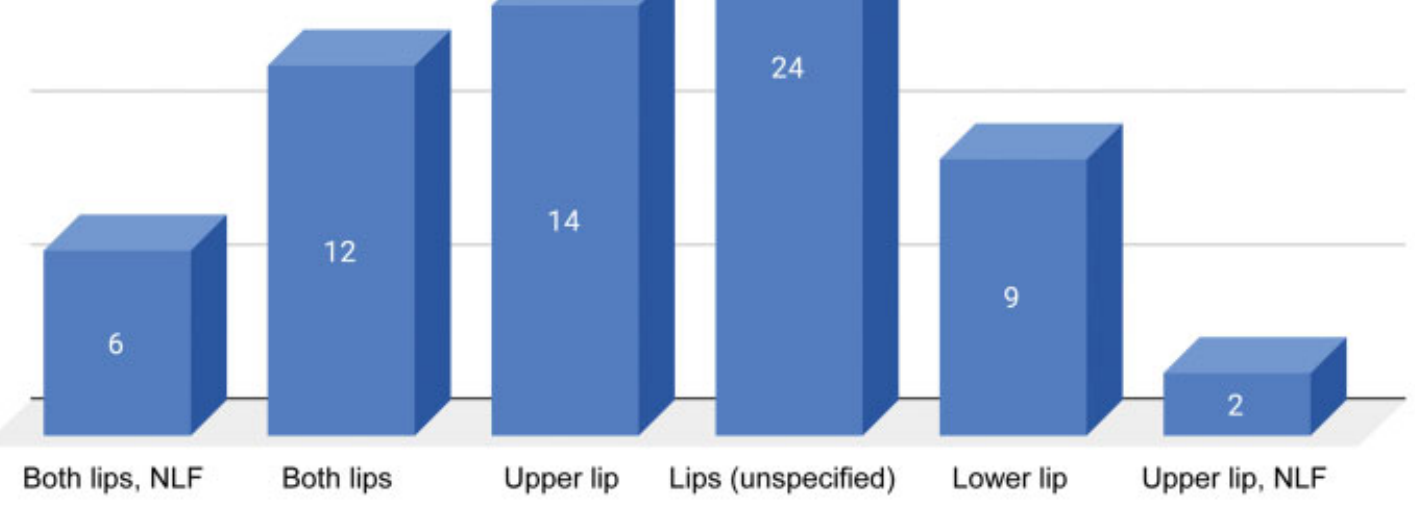

Fig. 4 Initial site of injection for augmentation.

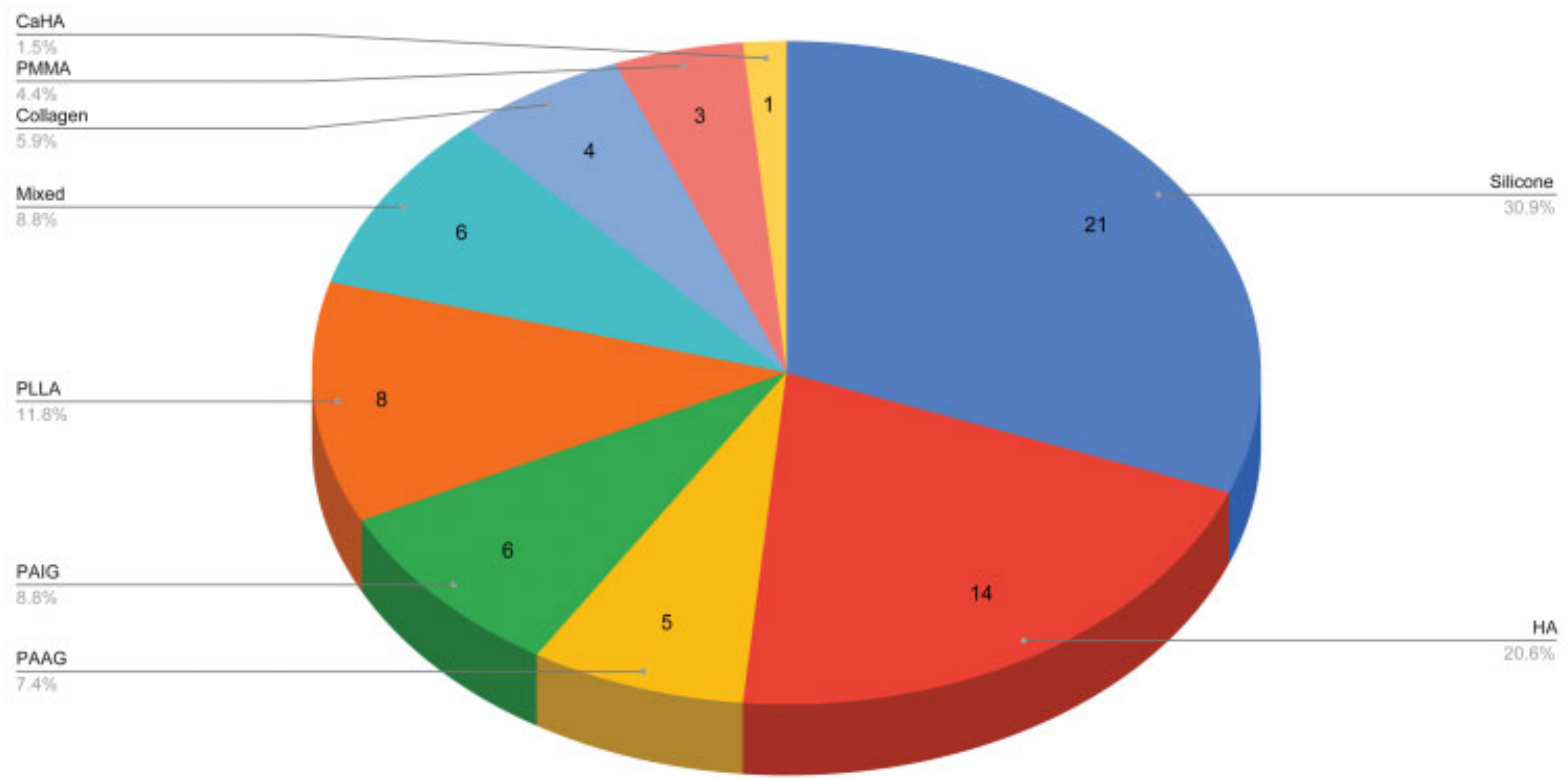

Fig. 5 Reported filler type used. CAHA, calcium hydroxy apatite; HA, hyaluronic acid; Mixed, combination of HA and hydroxy-ethyl-methacrylate (HEMA) and ethyl-methacrylate (EMA); PAAG, polyacrylamide gel; PAIG, polyalkylimide gel; PLLA, poly-L-lactic acid, PMMA, polymethylmethacrylate microspheres.

\section{Outcomes}

A summary of the outcomes is reported in - Table 3. In most cases, there was resolution of the nodule(s) or remission (42/68, 61.8\%). Eight cases reported only partial healing or persistent disease. Two cases reported a significant reduction in lesion size. Seventeen cases were lost to follow-up or did not report on the outcome.

\section{Discussion}

Lip augmentation with dermal fillers is rising in popularity. This systematic review analyzed reported cases of granulomas or nodules secondary to any dermal filler for lip augmentation. Of the 66 cases, 31 (47.0\%) confirmed the presence of a delayed-onset foreign-body 


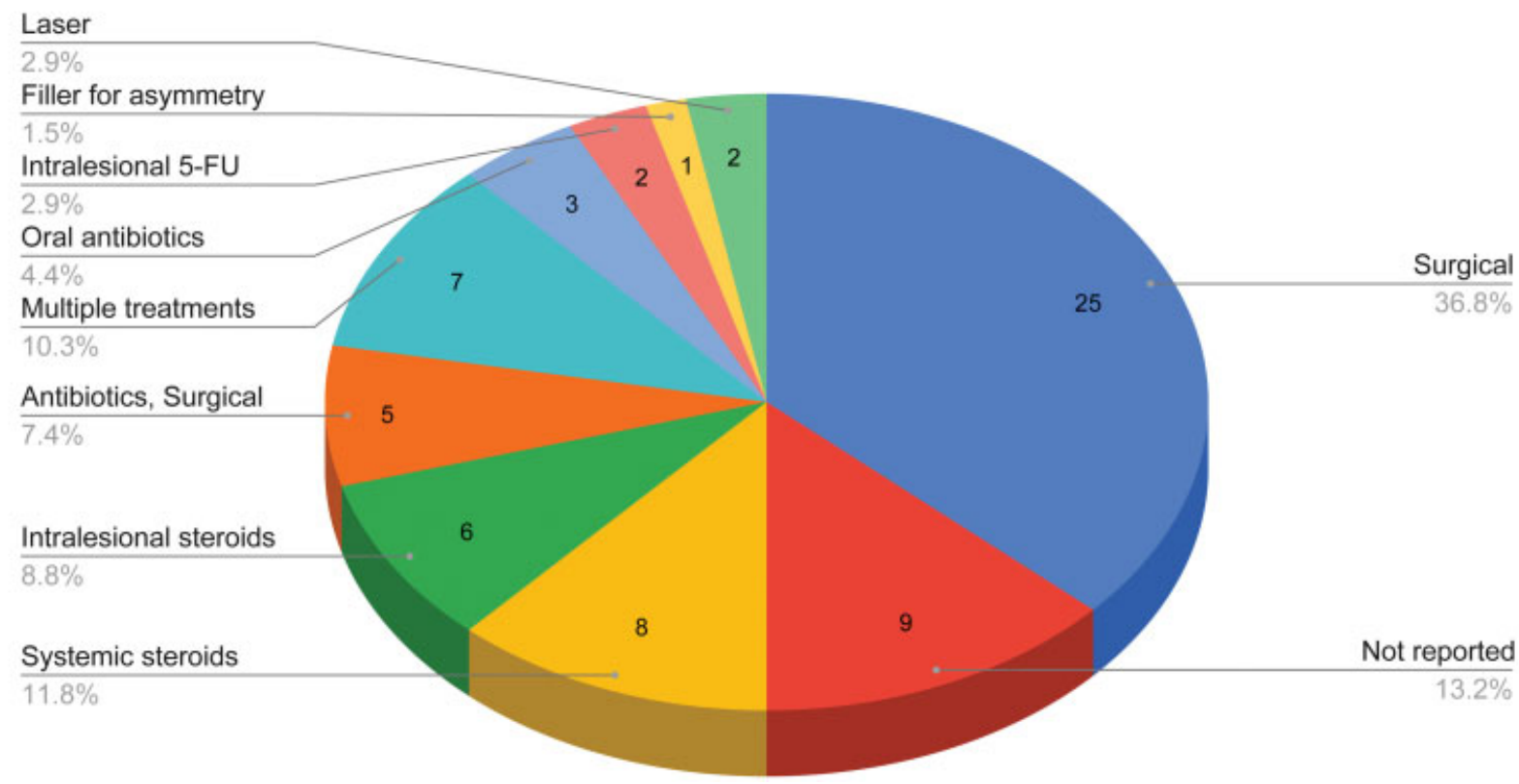

Fig. 6 Treatment of cases. FU, fluorouracil.

Table 3 Outcomes of reported cases

\begin{tabular}{|l|l|}
\hline Outcome & Number of cases (\%) \\
\hline Resolved or remission & $42(61.8 \%)$ \\
\hline $\begin{array}{l}\text { Incomplete resolution } \\
\text { (partial, persistent disease, } \\
\text { minor bouts) }\end{array}$ & $7(10.3 \%)$ \\
\hline $\begin{array}{l}\text { Significant reduction in lesion size } \\
\text { or progressive improvement }\end{array}$ & $3(4.4 \%)$ \\
\hline Lost to follow-up & $3(4.4 \%)$ \\
\hline Not reported & $13(19.1 \%)$ \\
\hline
\end{tabular}

granuloma. Of note, not all nodules are considered granulomas.

Nodules following treatment of filler are commonly categorized as inflammatory or noninflammatory in origin. ${ }^{13}$ Inflammatory nodules may occur days to years after treatment as a result of host response to a foreign body. In contrast, noninflammatory nodules commonly occur immediately after treatment and are typically caused by improper placement of the filler material. These cases may not be reported in the literature as observation, massage, or hyaluronidase may resolve these nodules. Temporary and biodegradable HA should have a minimal foreign-body response compared with permanent and nonresorbable fillers such as silicone. ${ }^{14}$ However, our study demonstrates that treatment with HA does not preclude a risk of granuloma formation. ${ }^{15}$

Diagnosis of lip nodules can be challenging as patients may not associate them with filler treatment performed weeks, months, or years prior. A broad range of differential diagnoses commonly includes abscesses, sialadenitis, mucocele, benign salivary gland neoplasm, or malignancy. Infection can present early or late in the clinical course and are more commonly single nodules. The involvement of multiple sites more likely suggests a foreign-body granulomatous response. Timely and proper diagnosis of these masses is crucial as they may mimic a neoplasm, which is particularly important given the generally older age group of these patients.

Silicone liquid (dimethylpolysiloxane) has been widely used for soft tissue augmentation. When it was first introduced in the late 1950s, it was considered safe as it was not known to elicit pathological disease in humans. Additionally, non-medical-grade silicone fluid was used in many patients. Years later, the term "siliconoma" was coined to describe the granulomatous reactions in soft tissues of patients who had received liquid silicone injections. ${ }^{16}$ The pathogenesis of silicone granuloma is unknown, but factors such as the volume of the injection, impurities present in the fillers, and the physical properties of fillers have been reported to affect granuloma formation. ${ }^{17}$ Silicone granulomas have been reported in other areas of the face such as the eyelids and cheek and the onset ranged from 5 months to 15 years, which is similar to that of our observed cases. ${ }^{18,19}$ Liquid silicone injections remain controversial, particularly in countries where there is inadequate control of quality of material used for soft tissue augmentation.

Several hypotheses have been suggested for the pathogenesis of granulomatous reactions to HA. HA is a polysaccharide that is fermented from bacteria and impurities from this process may elicit a hypersensitivity response, particularly in patients who have undergone repeated injections. ${ }^{15,20}$ Additionally, during the production of HA filler products, stabilization through a cross-linking process occurs, which allows the product to be resistant to natural hyaluronidases. Over time, the breakdown and byproducts of the cross-linked material may induce a host inflammatory response. ${ }^{21}$ Lastly, bacteria inoculated during the injection 
may form a biofilm. The biofilm surrounding the HA material creates a matrix that can inhibit natural hyaluronidases from degrading the HA. These biofilms can induce a minimal infection with little host response, making them asymptomatic for months or even years. ${ }^{22}$ In our cases, microscopic examination of the nodules confirmed the presence of HA years following treatment, indicating failure in the degradation process. A subsequent delayed foreign-body tissue reaction to biofilm could have been elicited in the months or years following initial injection.

Restylane was the most common HA filler used in our reported cases. One explanation of this finding may be due to the rheological properties (i.e., cohesion) or processing technologies of the fillers. Popular Restylane products for lip augmentation are non-animal HA, while Juvéderm uses Hylacross and Vycross cross-linking technologies. ${ }^{23}$ Bhojani-Lynch reported a case where two different brands (Teosyal Puresense Ultra Deep and Belotero Intense) were injected into various parts of the face during the same session and only areas treated with Teosyal triggered a hypersensitivity reaction characterized by diffuse redness and swelling without lumps. The authors suggest that reactions to HA fillers may be attributed to rheological or processing technologies of the fillers. ${ }^{24}$ Additionally, more reports of Restylane may be published as it was Food and Drug Administration-approved and more widely used earlier than Juvéderm and Belotero. In our cases, granuloma formation was more likely found in the upper lip compared with the lower lip, which may be due to the fact that the upper lips are more commonly treated. While our review includes studies involving granuloma formation following the use of calcium hydroxyl apatite and PLLA, current standard practice does not use these products.

Many patients were initially treated with nonoperative methods such as NSAIDs, antibiotics, and systematic and/or intralesional steroids. Laser therapy has also been successfully used. ${ }^{11}$ Goldman and Wollina reported the use of a subdermal, intralesional 1,064 nm neodymium-doped yttrium aluminum garnet (Nd:YAG) laser in combination with a blunt liposuction cannula suction in 81 patients with facial lumps or granulomas following PMMA. The procedure was well tolerated and $86.4 \%$ of the patients were satisfied. ${ }^{25}$ In our subset of patients, many ultimately required surgical removal of the granuloma, particularly if there was a single nodule. For those with multiple nodules, more aggressive treatments were pursued, including intralesional 5-FU.

\section{Limitations}

This systematic review has some limitations. The sample size is small with only 66 cases of dermal filler-related lip granuloma or nodules reported in the literature. There was also a lack of uniformity in describing the cases such as time of onset versus time the patient presented for care. There are many factors that determine the likelihood of dermal filler complications such as injector experience, training, and techniques used. However, this information was not available in any cases, potentially owing to the long duration from initial injection to time of onset of nodule(s). Some studies reporting on lip nodules or granulomas secondary in filler were excluded from our study because they did not provide enough patient case information. Therefore, our review may not have included all reported cases in the literature.

\section{Conclusion}

Understanding the sequelae of lip augmentation with dermal filler products allows clinicians to provide safe and effective treatment. Nodules that present months to years following filler treatment may represent a foreign-body granuloma. A combination of oral antibiotics, intralesional or oral steroids, and surgical excision successfully treated the majority of cases in our study. Future studies evaluating the development of granulomas should include treatment injection methods and techniques to better elucidate potentially related causes.

\section{Conflict of Interest}

None.

\section{References}

1 Hotta TA. Lip enhancement: physical assessment, injection techniques, and potential adverse events. Plast Surg Nurs 2018;38 (01):7-16

2 Luthra A. Shaping lips with fillers. J Cutan Aesthet Surg 2015;8 (03):139-142

3 Gupta A, Miller PJ. Management of lip complications. Facial Plast Surg Clin North Am 2019;27(04):565-570

4 Philipp-Dormston WG, Goodman GJ, De Boulle K, et al. Global approaches to the prevention and management of delayed-onset adverse reactions with hyaluronic acid-based fillers. Plast Reconstr Surg Glob Open 2020;8(04):e2730

5 Sadeghpour M, Quatrano NA, Bonati LM, Arndt KA, Dover JS, Kaminer MS. Delayed-onset nodules to differentially crosslinked hyaluronic acids: comparative incidence and risk assessment. Dermatol Surg 2019;45(08):1085-1094

6 Modarressi A, Nizet C, Lombardi T. Granulomas and nongranulomatous nodules after filler injection: different complications require different treatments. J Plast Reconstr Aesthet Surg 2020; 73(11):2010-2015

7 Dionyssopoulos A, Nikolis A, Patsatsi A, Sotiriadis D. Granulomas of the lips: a rare complication after injection of polylactic acid for aesthetic augmentation. J Plast Reconstr Aesthetic Surg 2007;60 (09):1079-1080

8 Alijotas-Reig J, Fernández-Figueras MT, Puig L. Pseudocystic encapsulation: a late noninflammatory complication of hyaluronic acid filler injections. Dermatol Surg 2013;39(11):1726-1728

9 Grippaudo FR, Di Girolamo M, Mattei M, Pucci E, Grippaudo C. Diagnosis and management of dermal filler complications in the perioral region. J Cosmet Laser Ther 2014;16(05):246-252

10 Curi MM, Cardoso CL, Curra C, Koga D, Benini MB. Late-onset adverse reactions related to hyaluronic acid dermal filler for aesthetic soft tissue augmentation. J Craniofac Surg 2015;26 (03):782-784

11 Goldman A, Wollina U. Polymethylmethacrylate-induced nodules of the lips: clinical presentation and management by intralesional neodymium:YAG laser therapy. Dermatol Ther (Heidelb) 2019;32 (01):e12755

12 Sanchis-Bielsa JM, Bagán JV, Poveda R, Salvador I. Foreign body granulomatous reactions to cosmetic fillers: a clinical study of 15 cases. Oral Surg Oral Med Oral Pathol Oral Radiol Endodontol 2009;108(02):237-241 
13 Ledon JA, Savas JA, Yang S, Franca K, Camacho I, Nouri K. Inflammatory nodules following soft tissue filler use: a review of causative agents, pathology and treatment options. Am J Clin Dermatol 2013;14(05):401-411

14 Christensen LH. Host tissue interaction, fate, and risks of degradable and nondegradable gel fillers. Dermatol Surg 2009;35 (Suppl 2):1612-1619

15 Rongioletti F, Atzori L, Ferreli C, et al. Granulomatous reactions after injections of multiple aesthetic micro-implants in temporal combinations: a complication of filler addiction. J Eur Acad Dermatol Venereol 2015;29(06):1188-1192

16 Ficarra G, Mosqueda-Taylor A, Carlos R. Silicone granuloma of the facial tissues: a report of seven cases. Oral Surg Oral Med Oral Pathol Oral Radiol Endod 2002;94(01):65-73

17 Lee JM, Kim YJ. Foreign body granulomas after the use of dermal fillers: pathophysiology, clinical appearance, histologic features, and treatment. Arch Plast Surg 2015;42(02):232-239

18 Chen TA, Mercado CL, Topping KL, Erickson BP, Cockerham KP, Kossler AL. Disseminated silicone granulomatosis in the face and orbit. Am J Ophthalmol Case Rep 2018;10:32-34

19 Requena C, Requena L, Alegre V, et al. Adverse reaction to silicone simulating orofacial granulomatosis. J Eur Acad Dermatol Venereol 2015;29(05):998-1001

20 Tonin B, Colato C, Bruni M, Girolomoni G, Affiliations MD. Late granuloma formation secondary to hyaluronic acid injection. Dermatol Online J 2020;26(07):13030/qt9fj4f3ts

21 Lupo MP. Hyaluronic acid fillers in facial rejuvenation. Semin Cutan Med Surg 2006;25(03):122-126

22 Marusza W, Mlynarczyk G, Olszanski R, et al. Probable biofilm formation in the cheek as a complication of soft tissue filler resulting from improper endodontic treatment of tooth 16 . Int J Nanomedicine 2012;7:1441-1447

23 Jiang B, Ramirez M, Ranjit-Reeves R, Baumann L, Woodward J. Noncollagen dermal fillers: a summary of the clinical trials used for their FDA approval. Dermatol Surg 2019;45(12): 1585-1596

24 Bhojani-Lynch T. Late-onset inflammatory response to hyaluronic acid dermal fillers. Plast Reconstr Surg Glob Open 2017;5(12): e1532

25 Goldman A, Wollina U. Intralesional neodymium YAG laser to treat complications of polymethylmethacrylate. Open Access Maced J Med Sci 2018;6(09):1636-1641

26 Kaczorowski M, Nelke K, Łuczak K, Hałoń A Filler migration and florid granulomatous reaction to hyaluronic acid mimicking a buccal tumor. J Craniofac Surg 2020;31(01):e78-e79

27 Alcântara CEP, Noronha MS, Cunha JF, Flores IL, Mesquita RA. Granulomatous reaction to hyaluronic acid filler material in oral and perioral region: a case report and review of literature. J Cosmet Dermatol 2018;17(04):578-583

28 Fernández-Aceñero MJ, Zamora E, Borbujo J. Granulomatous foreign body reaction against hyaluronic acid: report of a case after lip augmentation. Dermatol Surg 2003;29(12):1225-1226

29 Edwards PC, Fantasia JE, Iovino R. Foreign body reaction to hyaluronic acid (Restylane): an adverse outcome of lip augmen- tation. J Oral Maxillofac Surg 2006;64(08):1296-1299, discussion 1299

30 Farahani SS, Sexton J, Stone JD, Quinn K, Woo SB. Lip nodules caused by hyaluronic acid filler injection: report of three cases. Head Neck Pathol 2012;6(01):16-20

31 Park TH, Seo SW, Kim JK, Chang CH. Clinical experience with hyaluronic acid-filler complications. J Plast Reconstr Aesthet Surg 2011;64(07):892-896

32 Alghonaim YA, Solomon PD. Bilateral lower-lip foreign body granuloma secondary to hyaluronic acid injection. Plast Surg Case Stud 2016;2(02):16-17

33 Wang LL, Thomas WW, Ditre CM, Friedman O. Silicone granuloma formation associated with dermal injection. Ear Nose Throat J 2018;97(1-2):E52-E53

34 Schmidt-Westhausen AM, Frege J, Reichart PA. Abscess formation after lip augmentation with silicone: case report. Int J Oral Maxillofac Surg 2004;33(02):198-200

35 Hamilton DG, Gauthier N, Robertson BF. Late-onset, recurrent facial nodules associated with injection of poly-L-lactic acid. Dermatol Surg 2008;34(01):123-126, discussion 126

36 Dijkema SJ, van der Lei B, Kibbelaar RE. New-fill injections may induce late-onset foreign body granulomatous reaction. Plast Reconstr Surg 2005;115(05):76e-78e

37 Bigatà X, Ribera M, Bielsa I, Ferrándiz C. Adverse granulomatous reaction after cosmetic dermal silicone injection. Dermatol Surg 2001;27(02):198-200

38 Maly A, Regev E, Meir K, Maly B. Tissue reaction to liquid silicone simulating low-grade liposarcoma following lip augmentation. J Oral Pathol Med 2004;33(05):314

39 Alijotas-Reig J, Garcia-Gimenez V, Vilardell-Tarres M. Late-onset immune-mediated adverse effects after poly-L-lactic acid injection in non-HIV patients: clinical findings and long-term followup. Dermatology 2009;219(04):303-308

40 Akrish S, Dayan D, Taicher S, Adam I, Nagler RM. Foreign body granulomas after injection of Bio-alcamid for lip augmentation. Am J Otolaryngol 2009;30(05):356-359

41 Baumann LS, Halem ML. Lip silicone granulomatous foreign body reaction treated with aldara (imiquimod 5\%). Dermatol Surg 2003;29(04):429-432

42 da Costa Miguel MC, Nonaka CFW, dos Santos JN, Germano AR, de Souza LB. Oral foreign body granuloma: unusual presentation of a rare adverse reaction to permanent injectable cosmetic filler. Int J Oral Maxillofac Surg 2009;38(04):385-387

43 Friedmann DP, Kurian A, Fitzpatrick RE. Delayed granulomatous reactions to facial cosmetic injections of polymethylmethacrylate microspheres and liquid injectable silicone: a case series. J Cosmet Laser Ther 2016;18(03):170-173

44 Weyand B, Menke H. Case report: adverse granulomatous reaction (Granuloma formation) and pseudomonas superinfection after lip augmentation by the new filler DermaLive ${ }^{\circledR}$. Eur J Plast Surg 2008;30:291-295

45 Martin LHC, Hankinson PM, Khurram SA. Beauty is only mucosa deep: a retrospective analysis of oral lumps and bumps caused by cosmetic fillers. Br Dent J 2019;227(04):281-284 